\section{No evidence for heterozygote advantage at MTHFR in patients with lumbosacral myelomeningocele or their relatives}

To the Editor:

Weitkamp et al. ${ }^{1}$ reported a heterozygote advantage of the MTHFR gene in patients with neural tube defects (NTD) and their relatives. A C-to-T substitution at nucleotide 677 that converts an alanine to a valine residue in the 5,10-methylenetetrahydofolate-reductase gene (MTHFR) yields a common thermolabile variant (T) with $30 \%$ to $50 \%$ activity of the allelic form (C). Homozygosity for the C677T allele has been shown to be more prevalent in individuals with NTD and their parents, as compared to controls in several studies. ${ }^{2-5}$ Weitkamp et al. ${ }^{1}$ used a data sample of 168 families with NTD pregnancy (81 anencephaly, 1 encephalocele, and 86 spina bifida). The families were from a variety of ethnic origins consisting of 92.3\% Western European, 7.0\% Native American, 0.4\% African American, and $0.3 \%$ Asian. The study concluded that there was an excess of C/T heterozygosity in NTD affected females and also in fathers and unaffected brothers of NTD patients, demonstrating a C/T heterozygote advantage.

From our series of families collected as part of a national collaborative effort, we have identified 166 families in which the proband has lumbosacral myelomeningocele. Our sample includes 166 probands, 162 mothers, 136 fathers, and 120 unaffected sibs. These families were American Caucasian and had no history of neural tube defects before the birth of the proband. MTHFR was genotyped as previously reported. 6 Table 1 shows the number of offspring of each genotype according to sex and diagnosis. In some families, maternal (4 families) or paternal genotypes (30 families) were unavailable. We assigned these families to parental mating types assuming Hardy Weinberg probabilities. Expected values presented in Table 1 were calculated based on Mendelian probabilities. Chi-square tests were used to test for allelic and genotypic transmission distortion from Mendelian expectations in affected and unaffected offspring of heterozygous parents. Deviation from Hardy Weinberg Equilibrium (HWE) in parents of NTD probands was assessed. We also tested for deviation from HWE in NTD male and female offspring.

The allele transmission ratio from $\mathrm{C} / \mathrm{T}$ heterozygous parents was 84.93C: $86.67 \mathrm{~T}$ to offspring with NTD $\left(\chi^{2}=0.018, P=\right.$ $0.8932)$ and 55.27C: $65.7 \mathrm{~T}$ to unaffected offspring $\left(\chi^{2}=0.891, P\right.$ $=0.3451)$. Additionally, the number of offspring of each genotype $(\mathrm{C} / \mathrm{C}, \mathrm{C} / \mathrm{T}$, or $\mathrm{T} / \mathrm{T})$ among the 225.3 offspring of $\mathrm{C} / \mathrm{T}$ heterozygous parents did not differ from Mendelian expectations $\left(\chi^{2}\right.$ $=1.14, P=0.5655,2 \mathrm{df})$. This was the case in the 129.5 affected offspring $\left(\chi^{2}=1.0, P=0.61,2 \mathrm{df}\right)$, and the 95.76 unaffected offspring $\left(\chi^{2}=0.65, P=0.72,2 \mathrm{df}\right)$. Therefore, there is neither an excess of heterozygotes (107.22 observed, 112.65 expected) nor a deficiency of C/C (74.52 observed, 74.84 expected) or T/T homozygotes (43.56 observed, 37.82 expected).

There was no departure from Hardy-Weinberg equilibrium in mother's genotypes ( $65 \mathrm{C} / \mathrm{C}, 82 \mathrm{C} / \mathrm{T}, 20 \mathrm{~T} / \mathrm{T}$ observed; 67.05 C/C, 76.90 C/T, 22.05 T/T expected; $\left(\chi^{2}=0.71, P=0.70,2 \mathrm{df}\right)$ or father's genotypes $(63.91 \mathrm{C} / \mathrm{C}, 84.84 \mathrm{C} / \mathrm{T}, 17.25 \mathrm{~T} / \mathrm{T}$ observed; $68.11 \mathrm{C} / \mathrm{C}, 76.44 \mathrm{C} / \mathrm{T}, 21.45 \mathrm{~T} / \mathrm{T}$ expected; $\chi^{2}=2.0$, $P=0.3678,2 \mathrm{df})$. Weitkamp et al. ${ }^{1}$ found increased heterozygosity in affected female offspring compared to affected male

Table 1

Number of offspring of each genotype according to sex and diagnosis

\begin{tabular}{|c|c|c|c|c|c|c|c|c|c|c|c|c|c|c|c|}
\hline $\begin{array}{l}\text { Mating } \\
\text { type }\end{array}$ & $\begin{array}{c}\text { Estimated } \\
\text { \# of } \\
\text { matings }\end{array}$ & \multicolumn{14}{|c|}{ Number of offspring with genotype ${ }^{a}$} \\
\hline & & \multicolumn{7}{|c|}{ Affected } & \multicolumn{7}{|c|}{ Unaffected } \\
\hline \multirow[b]{2}{*}{ MAT PAT } & & \multicolumn{4}{|c|}{ Male } & \multicolumn{3}{|c|}{ Female } & \multicolumn{4}{|c|}{ Male } & \multicolumn{3}{|c|}{ Female } \\
\hline & & $\mathrm{CC}$ & $\mathrm{CT}$ & & TT & $\mathrm{CC}$ & $\mathrm{CT}$ & $\mathrm{TT}$ & $\mathrm{CC}$ & $\mathrm{CT}$ & & ГТ & $\mathrm{CC}$ & $\mathrm{CT}$ & $\mathrm{TT}$ \\
\hline CC TT & 6.08 & & 1.36 & & & & 4.72 & & 2 & & & & & 1 & \\
\hline TT CC & 5.98 & & 4.62 & 1 & & & & & & 2.2 & 0.54 & & & 1 & 0.82 \\
\hline $\mathrm{CC} \quad \mathrm{CT}$ & 29.76 & $10.9(8.5)$ & $6.1(8.5)$ & & & $10.3(8.8)$ & $7.3(8.8)$ & & $12.4(12.7)$ & $13 \quad(12.7)$ & & & $5.5(5.7)$ & $6 \quad(5.7)$ & \\
\hline $\mathrm{CT} \quad \mathrm{CC}$ & 29.23 & $5.1(5.8)$ & $6.6(5.8)$ & & & $9.1(8.8)$ & $8.5(8.8)$ & & $4 \quad(3.2)$ & $2.4(3.2)$ & & & $4 \quad(4.5)$ & $5 \quad(4.5)$ & \\
\hline CT $\mathrm{CT}$ & 42.06 & $3.4(4.8)$ & $10.7(9.5)$ & 5 & $(4.8)$ & $5.9(5.8)$ & $9.3(11.5)$ & $7.8(5.8)$ & $3 \quad(2.9)$ & $4.5(5.7)$ & 4 & (2.9) & $1 \quad(3.4)$ & $8.9(6.9)$ & $3.8 \quad(3.4)$ \\
\hline $\mathrm{CC}$ CC & 28.7 & 11.5 & 0.54 & & & 11.7 & & & 6.6 & & & & 10.5 & & \\
\hline CT $\mathrm{TT}$ & 10.63 & & $1.4(2.7)$ & 4 & $(2.7)$ & & $2.3(2.7)$ & $3.2(2.7)$ & & $1 \quad(2)$ & 3 & (2) & & $1.1(2.5)$ & $4 \quad(2.5)$ \\
\hline TT CT & 13.02 & & $3.4(3.6)$ & 3.8 & (3.6) & & $4 \quad(2.8)$ & $1.6(2.8)$ & & $2 \quad(2.2)$ & 2.5 & $(2.2)$ & & $4 \quad(2.4)$ & $0.82(2.4)$ \\
\hline TT $\mathrm{TT}$ & 0.54 & & & 0.18 & & & 0.36 & & & & & & & & 0.36 \\
\hline Total & 166 & $30.9(19.1)$ & $34.6(30.2) 1$ & & (11) & $37 \quad(23.3)$ & $36 \quad(34.6) 1$ & $13 \quad(11.3)$ & $28 \quad(18.7)$ & $25 \quad(25.8)$ & 10 & $(7.1)$ & $21 \quad(13.7)$ & $27 \quad(22)$ & $9.8 \quad(8.4)$ \\
\hline
\end{tabular}

${ }^{a}$ Expected values in parenthesis were based on Mendelian probabilities for gene transmission from heterozygous parents (or from all parents in the totals). Expected values in brackets were calculated assuming Hardy-Weinberg equilibrium for each group (spina bifida male, spina bifida female, unaffected male, unaffected female).

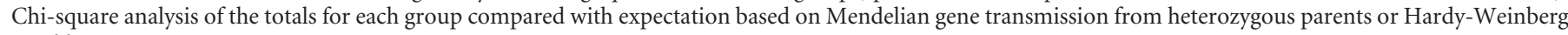
equilibrium. 
offspring, suggesting a sex difference. Because male fetuses may have a higher spontaneous abortion rate than females, this result may suggest that the MTHFR heterozygous genotype may reduce fetal survival in males. In our study, we failed to find any such evidence in affected females $(37 \mathrm{C} / \mathrm{C}, 36 \mathrm{C} / \mathrm{T}, 13 \mathrm{~T} / \mathrm{T}$ observed; 35.2 C/C, 39.7 C/T, 11.2 T/T expected; $\chi^{2}=0.72, P=0.70,2 \mathrm{df}$ ) or in affected males $(30.92 \mathrm{C} / \mathrm{C}, 34.64 \mathrm{C} / \mathrm{T}, 14 \mathrm{~T} / \mathrm{T}$ observed; 29.2 $\mathrm{C} / \mathrm{C}, 38 \mathrm{C} / \mathrm{T}, 12.3 \mathrm{~T} / \mathrm{T}$ expected; $\chi^{2}=0.632, P=0.73,2 \mathrm{df}$ ), or in the fathers $\left(68.11 \mathrm{C} / \mathrm{C}, 76.44 \mathrm{C} / \mathrm{T}, 21.45 \mathrm{~T} / \mathrm{T}\right.$ expected; $\chi^{2}=2.0, P$ $=0.37,2 \mathrm{df})$

There are several potential explanations for our failure to replicate the finding of Weitkamp et al. ${ }^{1}$ Firstly, it is well established that population stratification, known or unknown, can adversely impact the conclusions regarding allelic associations; in other words, the results found in the previous study may have been due purely to effects of mixing of different ethnic groups whereas our sample was limited to American Caucasians. Additionally, in several previous studies, an excess of females was generally reported among children with anencephaly, but no major difference was seen among spina bifida patients. ${ }^{7-9}$ Perhaps MTHFR heterozygous males may have a selective disadvantage in upper level NTDs, including anencephaly and not in lower level NTDs. This may have been the case in our sample, which consisted primarily of lumbosacral myelomeningocele NTDs.

\section{ACKNOWLEDGMENTS}

The authors gratefully acknowledge support from NS39818 and NS26630.

\section{References}

1. Weitkamp LR, Tackels DC, Hunter AG, Holmes LB, Schwartz CE. Heterozygote advantage of the MTHFR gene in patients with neural-tube defect and their relatives. Lancet 1998;351:1554-1555.

2. van der Put NM, Steegers-Theunissen RP, Frosst P, Trijbels FJ, Eskes TK, van den Heuvel LP et al. Mutated methylenetetrahydrofolate reductase as a risk factor for spina bifida. Lancet 1995;346:1070-1071.

3. Whitehead AS, Gallagherm P, Mills JL, Kirke P, Burke H, Molloy AM et al. A genetic defect in 5,10 methylenetetrahydrofolate reductase in neural tube defects. Quart J Med 1995;88:763-766.

4. Ou CY, Stevenson RE, Brown VK, Schwartz CE, Allen WP, Khoury MJ et al. 5,10 Methylenetetrahydrofolate reductase genetic polymorphism as a risk factor for neural tube defects. Am J Med Genet 1996;63:610-614.

5. Christensen B, Arbour L, Tran P, Leclerc D, Sabbaghian N, Platt R et al. Genetic polymorphisms in methylenetetrahydrofolate reductase and methionine synthase, folate levels in red blood cells, and risk of neural tube defects. Am J Med Genet 1999;84:151-157.

6. Rampersaud E, Melvin EC, Siegel D, Mehltretter L, Dickerson ME, George TM et al; NTD Collaborative. Updated investigations of the role of MTHFR in human neural tube defects. Clin Genet 2003;63:210-214.

7. Bale PM. Sacrococcygeal developmental abnormalities and tumors in children. Perspect Pediatr Pathol 1984;8:9-56.

8. Martinez Frias ML, Parralo JA, Salvador J, Frias JL. Sex ratios in neural tube defects. Lancet 1986;2:871-872.

9. Hall JG. Neural tube defects, sex ratios, and X inactivation. Lancet 1986;1334-1335:

E. Rampersaud, $M S P H$

C. Brusato, BS

E.C. Melvin, MS, CGC M.C. Speer, PhD

and the NTD Collaborative Group

Center for Human Genetics

Duke University Medical Center

Durham, North Carolina

K. Metcalf

NC School of Science and Math

Durham, North Carolina 